\title{
Comercio internacional de cítricos del NEA: competitividad en la Unión Europea
}

\author{
Molina ${ }^{1}$, Néstor A.; Taiariol ${ }^{1}$, Darío R. \\ ${ }^{1}$ INTA EEA Bella Vista. \\ Correo electrónico:nmolina@correo.inta.gov.ar
}

\begin{abstract}
Resumen
Se estudia el comportamiento de las exportaciones de naranjas frescas argentinas a la Unión Europea, respecto de España, Uruguay y Sudáfrica. El origen de la oferta nacional indica a Entre Ríos como la principal provincia exportadora. En segundo lugar se establece la producción mundial de naranjas que alcanzó 68.5 millones de toneladas (2009), el comercio internacional es de 5.2 millones $(97,7 \%$ con destino al hemisferio norte); el mayor destino es la UE (2.8 millones). Los principales abastecedores son España (32.8\%) y Argentina, Uruguay y Sudáfrica (20,0\%) a contraestación. A continuación se analiza el índice de ventaja comparativa revelada (VCR) y el análisis de participación constante en las exportaciones mundiales. La participación de Argentina en las últimas dos décadas promedió el 3.6\%, siendo sus principales mercados en este período Holanda, España, Alemania, Bélgica y Reino Unido. Se propone una estrategia de complementación entre Argentina y Uruguay para competir con Sudáfrica en la Unión Europea.
\end{abstract}

Palabras clave: naranja, competitividad, comercio, Argentina

\section{Summary}

We study the behavior of Argentine exports of fresh oranges to the EU, with respect to Spain, Uruguay and South Africa. The domestic supply source tells Entre Rios province as the main exporter. Second set the world production of oranges which reached 68.5 million tons (2009), international trade is 5.2 million ( $97.7 \%$ are for the northern hemisphere), the main destination is the EU (2.8 million). The main suppliers are Spain (32.8\%) and Argentina, Uruguay and South Africa $(20.0 \%)$ to counter season. Then he examines the revealed comparative advantage index (RCA) and analysis of ongoing participation in world exports. The participation of Argentina in the last two decades has averaged $3.6 \%$, with its main markets in this period the Netherlands, Spain, Germany, Belgium and the UK. We propose a strategy of complementation between Argentina and Uruguay to compete with South Africa in the European Union.

Keywords: orange, competitiveness, trade, Argentina

\section{INTRODUCCIÓN}

La Unión Europea es el principal importador mundial de naranja fresca, con una población de quinientos millones de habitantes y un consumo per capita de $11.5 \mathrm{Kg}$, importó durante el 20092.8 millones de toneladas. Entre los principales proveedores se encuentran España, Marruecos, Uruguay y Sudáfrica; que en el período 2001-2009, promediaron el 78.9\% del volumen aprovisionado.

La producción de naranjas a nivel mundial alcanzó las 64 millones de toneladas (FAOSTAT, 2011), de las cuales Argentina produce el 1.1\% y comercializa a contraestación a la Unión Europea. El origen de la producción nacional se encuentra en la Región del Nordeste (con el $76 \%$ ) y el calendario de oferta va de mayo a noviembre (Federcitrus, 2011)

La competitividad del las exportaciones de naranja fresca argentina deben ser comparadas con otros competidores a efectos de mejorar el comportamien- to exportador de las regiones productores. Entre los competidores analizamos: España (es miembro de la Unión Europea y principal proveedor de naranjas, creció de $46.7 \%$ al $53.7 \%$ en las últimas dos décadas), Marruecos (forma parte del grupo de países del Mediterráneo y es el principal exportador entre los proveedores no miembros del hemisferio norte) y del hemisferio sur, Sudáfrica, Argentina y Uruguay suman el $20,0 \%$ de las importaciones.

El objetivo de nuestra investigación es conocer la competitividad de las exportaciones de naranjas frescas argentinas a la Unión Europea y la situación de los principales proveedores.

\section{MATERIALES Y MÉTODOS}

Una vez descripta la situación productiva en nuestro país, pasamos a establecer la actualidad del comercio de naranja fresca en la Unión Europea, mercado principal de alimentos frescos del mundo. En 
lo que acontece al análisis de competitividad; nos interesa definir el régimen de importación de la Unión Europea con los países definidos (competitividad aduanera), para pasar a establecer los efectos estructurales, de competitividad e interacción en dos niveles de descomposición de las exportaciones de acuerdo al análisis de la participación constante en el mercado (competitividad estadística para el período 1991 - 2009).

Situación en Argentina. La producción Argentina de cítricos se encuentra en el orden de los 2,6 millones de t. en 135 mil hectáreas, la naranja equivale al 32,6\% de este volumen. Según la Federación Argentina del Citrus (2011 a), la producción de naranja se encuentra diferenciada en dos regiones: Nordeste Argentino (NEA) que en la campaña 2010 produjo el $76,1 \%$ concentrándose la producción en las provincias de Entre Ríos (56.6\%) y Corrientes (15.6\%) y el Noroeste Argentino (NOA) con el $23.9 \%$. El cronograma de producción que permite la oferta nacional de naranja indica que la región NEA produce en forma creciente hasta el final de año (Zubrzycki, H., 1997).

Nuestro país representa el $4,3 \%$ de las exportaciones de naranja fresca a nivel mundial, el 6,0\% de mandarina y 17,6\% de limón. Según la Oficina de Comercio Exterior del SENASA, durante la campaña 2010 se exportó 162 mil toneladas desde Argentina, de este volumen 51,9\% corresponde a la Unión Europea, el período de ingreso a este mercado es junio - septiembre con el 93,3\% del volumen exportado a este mercado.

En el Nordeste Argentino, el período de cosecha se presenta en Misiones entre agosto y octubre con el $88 \%$ de la producción, Corrientes tiene producción tardía puesto que el $87 \%$ se da entre septiembre y noviembre, en cambio Entre Ríos tiene una amplitud de oferta entre septiembre y enero, llegando a dos tercios de la producción. Por último, Buenos Aires se caracteriza por tener una oferta temprana, relacionada con el trimestre agosto - octubre, con el 69\% (Molina, 2003)

Según el SENASA (2011), se exportaron durante 2010, 83.253 t., siendo las principales provincias exportadoras: Entre Ríos (40,3\%) y Corrientes (9,2\%). La oferta de naranja argentina permite seguir exportando con posterioridad al pico de exportación, el período disponible para exportar es junio a septiembre con precios que van de U\$S 498 a U\$S452 por tonelada. (USDA 2010)

Exportaciones a la Unión Europea. La producción mundial de cítricos se duplicó en los últimos 30 años, pasando de 52.5 millones de t. en 1979 a 116,6 millones en 2009, la naranja representan el 60\% de la oferta mundial de cítricos (FAOSTAT, 2011), en la última década creció 5 millones de t. pasando de 58.9 a 64.1 millones.
Tabla 1. Producción de naranjas según hemisferios y principales países. En millones de t.

\begin{tabular}{|c|c|c|c|}
\hline & Década 1980 & Década 1990 & 2001-2009 \\
\hline Total Mundial & 43,2 & 58,9 & 64,4 \\
\hline Hemisferio Norte & 26,3 & 34,9 & 42,1 \\
\hline EEUU & 7,4 & 10,0 & 9,5 \\
\hline Unión Europea & 4,9 & 5,6 & 6,2 \\
\hline España & 2,1 & 2,6 & 2,9 \\
\hline Grecia & 1,9 & 1,8 & 2,1 \\
\hline Italia & 0,8 & 0,9 & 0,9 \\
\hline Mediterráneo & 4,2 & 4,7 & 5,8 \\
\hline Hemisferio Sur & 16,9 & 24,0 & 22,2 \\
\hline Sudáfrica & 0,6 & 0,9 & 1,4 \\
\hline Argentina & 0,6 & 0,8 & 0,8 \\
\hline Uruguay & 0,1 & 0,1 & 0,1 \\
\hline Brasil & 14,0 & 20,4 & 17,9 \\
\hline
\end{tabular}

Fuente: FAOSTAT (2011) Nota: Mediterráneo está compuesto por Argelia, Egipto, Israel, Jordania, Líbano, Marruecos, Palestina, Siria, Túnez y Turquía.

El hemisferio sur participó con el 39\% de la oferta mundial en la década del 80 , al $32 \%$ en la última década (Tabla 1). En la campaña 2009, Brasil es el principal productor del mundo con 18 millones de t., Estados Unidos en $2^{\circ}$ lugar, Sudáfrica figura $13^{\circ}$ lugar y Argentina, $16^{\circ}$.

El comercio internacional en los países productores en 2009 alcanzó a 5.7 millones t. de naranja, correspondiendo al hemisferio norte el $75.5 \%$; siendo el mayor importador la Unión Europea con $2.8 \mathrm{mi}-$ llones. Además, el comercio intracomunitario con origen en los países del sur del bloque como España, Italia, Grecia, Portugal y Francia representan $1.6 \mathrm{mi}-$ llones de toneladas, de las cuales un $84.7 \%$ se originan en España.

Los países exportadores no miembros se dividen en proveedores del hemisferio norte y del sur, caracterizados por tener distintas temporadas de exportación de naranja. Los países del cono sur son complementarios en la producción y comercio de cítricos, con lo cual es necesario desarrollar esta oportunidad. Nuestras exportaciones son en "contraestación" (período junio - septiembre) con el Hemisferio Norte.

En la campaña 2010/2011, la UE produjo 6,11 millones de t. e importó 1,2 millones de las cuales se destinó a consumo en fresco 5.834.000 de toneladas (USDA 2010c), con una población de 499,7 millones da un consumo anual de $11.5 \mathrm{~kg} /$ capita. Los principales abastecedores en el hemisferio norte; los países miembros España, Italia y Grecia, países del Mediterráneo y a contraestación en el hemisferio sur, Argentina, Uruguay y Sudáfrica.

En este artículo revisa la literatura económica internacional sobre índices de competitividad interna- 
cional: 1) ventaja comparativa revelada y 2) análisis de participación constante de mercado. Estas herramientas cuantitativas exigen como insumo los datos de exportación de un país en un determinado período de tiempo, y necesita de información secundaria provista por las bases de datos: FAOSTAT, COMTRADE, SENASA y Trade Map.

El índice de ventaja comparativa revelada (VCR) permite conocer la especialización exportadora de un país. Se define como el cociente entre la participación de un producto en las exportaciones de un país y la participación de ese mismo producto en las exportaciones mundiales. Un valor mayor (menor) a uno indica la presencia (ausencia) de VCR en ese producto porque, en relación al total exportado, exporta más que el mundo (Porto, 2010).

La fórmula propuesta por Balassa es:

$$
V C R a, c V C R a, c_{i}=\frac{X a, c_{i} / X a, t}{X w, c_{i} / X w, t}
$$

Donde: Xa,ci: exportaciones argentinas de naranja; Xa,t: exportaciones totales argentinas; Xw,ci: exportaciones mundiales de naranja y Xw,t: exportaciones totales mundiales.

El Análisis de Participación Constante es una técnica estadística utilizada para conocer el cambio en la comercialización de un producto de un país hacia otro países o países en un período de tiempo. Se trata de descomponer el crecimiento de las exportaciones y estudiar su comportamiento para evaluar si el cambio se corresponde con factores estructurales o la competitividad del sector bajo análisis (García Andía, 2000). El objetivo es averiguar hasta qué punto la especialización de un país en la exportación a determinados mercados disminuye o aumenta su oferta al mercado mundial.

Empezamos indicando el cambio total de las exportaciones de cítricos de argentina a la Unión Europea durante el período 2006-2009 versus el período 1991-1995 $(\Delta q)$ es el porcentaje de participación del país $\left(\sigma^{1}\right.$ y $\left.\sigma^{0}\right)$ en los períodos mencionados en el volumen importado por este bloque económico $\left(X^{1}\right.$ y $\left.X^{0}\right)$.

$\Delta q=\left(\sigma^{1} X^{1}-\sigma^{0} X^{0}\right)$

Es la suma o primera descomposición

De la sumatoria de los efectos estructural, competitividad e interacción de los anteriores. Siendo el efecto estructural (EE) el cambio esperado en las exportaciones, si se mantiene constante la participación inicial de Argentina en ese mercado:

(1.0) $\mathrm{EE}=\sigma^{0}\left(X^{1}-X^{0}\right)$

Si es positivo, el crecimiento de la demanda de cítricos afecta positivamente el crecimiento de las exportaciones.

El efecto competitividad es el cambio en las exportaciones atribuido a cambios en la competitividad ocurrida en ese periodo.
(2.0) $\mathrm{EX}=X^{0}\left(\sigma^{1}-\sigma^{0}\right)$

Si es positivo, el país gana competitividad.

Por último, por una cuestión matemática se incorpora el efecto interacción (EI); que mide la influencia de los cambios en la participación de mercado con los cambios en la demanda.

(3.0) $\mathrm{EI}=\left(\sigma^{1}-\sigma^{0}\right)\left(X^{1}-X^{0}\right)$

En un segundo nivel de descomposición cada uno de estos tres efectos se desagrega en dos obteniéndose seis efectos;

El efecto estructural se descompone en los efectos crecimiento y mercado:

El efecto crecimiento (EC) mide el crecimiento de las exportaciones atribuido a la demanda mundial por ese producto $\left(S^{0}\right)$, cuando analizamos por país de destino nos referimos a la Unión Europea. Es el cambio hipotético en las exportaciones, que podría haber ocurrido si la participación de un exportador en el mercado mundial se hubiera mantenido constante durante el periodo inicial $\left(S^{0}\right)$.

(4.1) $\mathrm{EC}=S^{0}\left(X^{1}-X^{0}\right)$

Efecto mercado (EM) es el cambio adicional esperado en las exportaciones, si el exportador mantiene su participación inicial en el mercado de la Unión Europea durante el periodo.

(5.1) $\mathrm{EM}=\left(\sigma^{0}-S^{0}\right)\left(X^{1}-X^{0}\right)$

Si es positivo indica que el país en cuestión tiende a concentrar sus exportaciones en mercados que crecen rápidamente.

El efecto competitividad se descompone en general (ECG) y específico (ECE):

Efecto competitivo general es la parte del cambio hipotético en las exportaciones, atribuible a cambios en la competitividad en general.

(6.2) $\mathrm{ECG}=X^{0}\left(S^{1}-S^{0}\right)$

Efecto competitivo específico representa la parte del cambio hipotético en las exportaciones, atribuible a cambios en la competitividad específica del mercado de la Unión Europea

(7.2) $\mathrm{ECE}=X^{0}\left(\sigma^{1}-\sigma^{0}+S^{0}-S^{1}\right)$

$\mathrm{El}$ efecto interacción se divide en puro (EIP) y residual (ER):

El efecto de interacción puro mide la interacción entre los cambios de la participación de un exportador en el mercado del país de destino y los cambios en el nivel de la Unión Europea.

(8.3) $\mathrm{EIP}=\left(\frac{X^{1}}{X^{0}}-1\right) X^{0}\left(S^{1}-S^{0}\right)$

Y, por último, el efecto residual mide la interacción entre los cambios de la participación de un exportador en el mercado del país de destino y los cambios en el nivel de demanda de éste.

(9.3) $\mathrm{ER}=\left(\sigma^{1}-\sigma^{0}\right)\left(X^{1}-X^{0}\right)-\left(\frac{X^{1}}{X^{0}}-1\right) X^{0}\left(S^{1}-S^{0}\right)$ 


\section{RESULTADOS Y DISCUSIÓN}

Como surge de la Tabla 2, los coeficientes asumen para todos los años valores mayores que uno, y siempre en mejor situación la naranja, lo que revela la existencia de un desempeño, tanto para el agregado como para cada especie cítrica, por arriba del promedio de las exportaciones mundiales. En nuestro caso, da 8.2 en el 2006 y crece hasta alcanzar el nivel de 11.2 en el 2010

Aplicando el método estadístico de participación constante del mercado, se realizó un ejercicio para determinar el nivel de competitividad que guardan las naranjas de exportación argentinas en el mercado de la Unión Europea frente a sus principales rivales, identificándose entre éstos a Sudáfrica, España, Uruguay y Marruecos. Y posteriormente, se repitió el análisis para Argentina y sus principales clientes en el bloque europeo (Tabla 3).

Como se observa en el cuadro anterior, Argentina representa el 3.8\% de las exportaciones de naranja a la Unión Europea durante la década 2000 - 2009, Sudáfrica $16.2 \%$, España $53.1 \%$, Uruguay $2.4 \%$ y Marruecos $6.5 \%$, totalizando el $82 \%$. En el período
1991-2009, Sudáfrica duplicó su participación del $8.4 \%$ al $17.3 \%$ y Marruecos disminuyó del $19.4 \%$ al $4.1 \%$, los demás países se mantuvieron estables.

Por otra parte, el efecto estructural fue positivo para los cinco países, incluyendo Marruecos, indicando que el efecto de la demanda de naranjas frescas fue positivo en el crecimiento de las exportaciones de los países proveedores.

Respecto a la competitividad, Sudáfrica es el país que registra el mayor crecimiento de la misma ${ }^{1}$, seguido por Argentina. El efecto competitividad es negativo para Marruecos, España y Uruguay, significando la pérdida de este factor en el mercado europeo.

El efecto interacción es también positivo para dos países (Argentina y Sudáfrica), definiendo que la interacción de cambios entre la participación de mercado y en la demanda los ha beneficiado, siendo negativo para el resto.

El efecto crecimiento que es atribuido al aumento de la demanda mundial por el producto, es positivo Sudáfrica, sustancialmente por encima de Argentina y el resto de competidores.

Tabla 2. Índice de Ventaja Común Revelada. Período 2006/2010

\begin{tabular}{|c|c|c|c|c|c|}
\hline & 2006 & 2007 & 2.008 & 2009 & 2010 \\
\hline \multicolumn{6}{|c|}{ Exportaciones de Naranja (en dólares) } \\
\hline España & 2.747 .322 & 3.364 .182 & 3.571 .830 & 3.449 .744 & 3.391 .357 \\
\hline Marruecos & 269.210 & 267.062 & 420.730 & 280.916 & 605.539 \\
\hline Argentina & 234.645 & 325.740 & 553.384 & 302.945 & 564.981 \\
\hline Uruguay & 60.696 & 74.157 & 76.226 & 71.069 & 87.352 \\
\hline Sudáfrica & 495.039 & 607.096 & 663.344 & 631.099 & 896.913 \\
\hline Mundo & 7.355 .944 & 8.856 .657 & 10.418 .828 & 10.048 .270 & 11.546 .982 \\
\hline \multicolumn{6}{|c|}{ Exportaciones totales (en dólares) } \\
\hline España & 214.061.202 & 253.753 .922 & 279.231 .468 & 223.132 .208 & 246.043 .652 \\
\hline Marruecos & 12.530 .624 & 14.607.346 & 20.305 .698 & 13.987 .850 & 17.716.674 \\
\hline Argentina & 46.546 .224 & 55.779 .580 & 70.015 .857 & 55.669 .065 & 62.929 .171 \\
\hline Uruguay & 3.952 .321 & 4.517 .549 & 5.941 .895 & 5.385 .509 & 6.941 .906 \\
\hline Sudáfrica & 52.601 .760 & 64.026 .608 & 73.965 .546 & 53.863 .892 & 71.484 .309 \\
\hline Mundo & 12.013.917.899 & 13.843.138.110 & 15.981.789.284 & 12.318.898.161 & 14.464 .843 .887 \\
\hline \multicolumn{6}{|c|}{ Índice de Ventaja Común Revelada } \\
\hline España & 21,0 & 20,7 & 19,6 & 19,0 & 17,3 \\
\hline Marruecos & 35,1 & 28,6 & 31,8 & 24,6 & 42,8 \\
\hline Argentina & 8,2 & 9,1 & 12,1 & 6,7 & 11,2 \\
\hline Uruguay & 25,1 & 25,7 & 19,7 & 16,2 & 15,8 \\
\hline Sudáfrica & 15,4 & 14,8 & 13,8 & 14,4 & 15,7 \\
\hline
\end{tabular}

Fuente: Trademap (2011) (http://www.trademap.org/tradestat/Country_SelProduct_TS.aspx)

1 Ndou y Obi (2011) en un reciente trabajo estiman que la naranja sudafricana gana competitividad por cuestiones del entorno del sector, como la desregulación del sector frutícola, la reforma agraria, los factores macroeconómicos y las normas de seguridad alimentaria global. Entonces, los factores relacionados con el crecimiento del mercado de exportación, en relación al crecimiento de las exportaciones mundiales (efecto estructural), las mejoras en la competitividad del país exportador (efecto competencia) y el efecto interacción. 
Tabla 3. Descomposición del cambio de exportaciones a la Unión Europea

\begin{tabular}{lcrrrr}
\hline UNION EUROPEA & Argentina & Sudáfrica & España & Uruguay & Marruecos \\
\hline Cambio en las exportaciones. & & & & & \\
Promedio 2006-2009 & 103.045 & 446.478 & 1.267 .950 & 64.484 & 127.252 \\
Promedio 1991-1995 & 69.918 & 184.912 & 1.034 .744 & 53.274 & 288.763 \\
$\Delta q=\left(\sigma^{1} X^{1}-\sigma^{0} X^{0}\right)$ & 33.127 & 261.566 & 233.206 & 11.210 & -161.511 \\
\hline Primer nivel de descomposición del cambio en las exportaciones & & & & \\
1. Efecto estructural (EE) & 18.203 & 48.142 & 269.396 & 13.870 & 75.180 \\
2. Efecto competitividad (EX) & 11.841 & 169.337 & -28.714 & -2.110 & -187.798 \\
3. Efecto interacción (EI) & 3.083 & 44.087 & -7.476 & -549 & -48.893 \\
\hline Segundo nivel de descomposición del cambio en las exportaciones & & & & \\
4. Efecto Crecimiento (EC) & 27.558 & 412.077 & -229.415 & -14.549 & -120.908 \\
5. Efecto Mercado (EM) & -9.354 & -363.935 & 498.811 & 28.418 & 196.087 \\
6. Efecto Competitivo General (ECG) & 11.295 & 316.385 & -365.961 & -22.056 & -145.060 \\
7. Efecto Competitivo Específico (ECE) & 545 & -147.048 & 337.247 & 19.946 & -42.738 \\
8. Efecto Interacción Pura (EIP) & 2.941 & 82.371 & -95.278 & -5.742 & -37.766 \\
9. Efecto Residual (ER) & 142 & -38.284 & 87.802 & 5.193 & -11.127 \\
\hline
\end{tabular}

Tabla 4. Descomposición del cambio de exportaciones de Argentina a países seleccionados

\begin{tabular}{lcrrrr}
\hline ARGENTINA & Holanda & España & Reino Unido & \multicolumn{1}{c}{ Francia } & Alemania \\
\hline Promedio 2006-2009 & 40.571 & 1.387 & 8.906 & 12.133 & 5.854 \\
Promedio 1991-1995 & 33.206 & 26.295 & 11.334 & 4.600 & 6.286 \\
$\Delta q=\left(\sigma^{1} X^{1}-\sigma^{0} X^{0}\right)$ & -7.365 & 24.908 & 2.427 & -7.533 & 431 \\
\hline Primer nivel de descomposición del cambio en las exportaciones & & & & \\
1. Efecto estructural (EE) & 5.230 & 21.092 & -137 & -2.395 & -273 \\
2. Efecto competitividad (EX) & -14.453 & 235 & 2.605 & -6.402 & 739 \\
3. Efecto interacción (EI) & 1.858 & 3.580 & -40 & 1.264 & -34 \\
\hline Segundo nivel de descomposición del cambio en las exportaciones & & & & \\
4. Efecto Crecimiento (EC) & 4.147 & 5.281 & 1.562 & -1.056 & 2.193 \\
5. Efecto Mercado (EM) & 1.083 & 15.811 & -1.699 & -1.339 & -2.466 \\
6. Efecto Competitivo General (ECG) & 2.194 & 50 & 1.748 & 2.910 & 3.235 \\
7. Efecto Competitivo Específico (ECE) & -16.647 & 186 & 857 & -9.312 & -2.496 \\
8. Efecto Interacción Pura (EIP) & 283 & 758 & -27 & -574 & -151 \\
9. Efecto Residual (ER) & 1.575 & 2.822 & -13 & 1.838 & 116 \\
\hline
\end{tabular}

Por otra parte, el efecto mercado que representa el cambio adicional en las exportaciones, si se mantiene constante la participación en el mercado europeo, es favorable para España, Marruecos y en menor medida Uruguay, indicando una concentración de exportaciones en este mercado; y es negativo para Sudáfrica y Argentina, señalando la cercanía de los dos primeros países al mercado de destino.

El efecto competitivo general, positivo sólo para Sudáfrica y Argentina, muestra que estos países han incrementado su nivel de competitividad en general y no así el resto. Por otra parte, el efecto competitivo específico que señala cambios en las exportaciones, atribuibles a cambios en la competitividad específica del mercado europeo, es positivo para España, y en menor medida Uruguay y Argentina; es decir, España ganó competitividad en el mercado de la UE.

El efecto interacción pura, que mide la interacción entre los cambios de la participación de un exportador en el mercado de la UE y los cambios en el nivel de la demanda mundial, es positivo para Sudáfrica y Argentina solamente; y el efecto residual, que señala la interacción positiva de estos exportadores en el mercado europeo y el cambio en el nivel de demanda del mismo país, es positivo para España y Uruguay. Estos países responden afirmativamente a la dinámica de la demanda del mercado mundial, y el europeo.

De esta manera se puede considerar que España y Sudáfrica, son los países que mejor se adaptan a 
la dinámica de la demanda del mercado de la Unión Europea.

Como se observa en la Tabla 4, Argentina representa el $19.7 \%$ de las importaciones de España, el $11 \%$ de Holanda, y en menor medida Reino Unido, Francia y Alemania. El efecto estructural fue positivo en España y Holanda. Respecto a la competitividad, el Reino Unido es el país de mejor ubicación. El efecto interacción es positivo para España, Holanda y Francia.

El efecto crecimiento es positivo para todos los países, menos Francia. Y el efecto mercado es importante en España y Holanda. En síntesis, el mercado europeo de mejor comportamiento para las naranjas argentinas es España.

\section{CONCLUSIONES}

Las competitividades pueden ser definidas por los siguientes ítems:

Según campaña de marketing, comparte su temporada con Uruguay y en mayo (al inicio de la temporada de exportación) compite con las naranjas del sur de Europa, especialmente España y Marruecos y en noviembre (final de la campaña) con España, Marruecos y Sudáfrica. En especial, dispone de un período más extenso que Sudáfrica, entonces el cronograma de comercio exterior se acota a los meses de junio a octubre, en ese período está disponible el $52.1 \%$ de la producción nacional.

Con respecto a los índices de competitividad: la ventaja comparativa revelada, que permite conocer la especialización exportadora durante el período 1996/2010; Argentina tuvo el peor comportamiento con 9.5, le siguió Sudáfrica con 14,8, España 19.5, Uruguay 20,5 y Marruecos es el índice más alto con 32.6.

$\mathrm{Y}$, por último, el análisis de participación constante de mercado nos permite conocer el cambio en la comercialización de un producto de un país hacia otro países o países en un período de tiempo. Los principales efectos arrojan los siguientes datos:

Efecto estructural: fue positivo para los cinco países, lo que indica que el efecto de la demanda de naranjas frescas fue positivo en el crecimiento de las exportaciones de los países proveedores.

Efecto competitividad: Sudáfrica registra el mayor crecimiento, seguido por Argentina. El efecto competitividad es negativo para Marruecos, España y Uruguay, significando la pérdida de este factor en el mercado europeo.

Efecto interacción; es positivo para Argentina y Sudáfrica, porque la interacción de cambios entre la participación de mercado y en la demanda los ha beneficiado, siendo negativo para el resto.

\section{BIBLIOGRAFIA}

FEDERCITRUS (2011) La actividad citrícola argentina. Año 2010. Federación Argentina del Citrus. Buenos Aires. 18 p. www.federcitrus.org.ar/actividad-citricola-2011.pdf Acceso a la página, Junio de 2011

Gracia Andía, A. (2000) El análisis de cuotas de mercado constantes en la literatura económica internacional. Universidad de Zaragoza. España 18 p. http:// www.unizar.es/centros/eues/html/archivos/temporales/10_AIS/AIS_10_10.pdf

Molina, N. (2003): Formulación de un plan de exportación citrícola. INTA EEA Bella Vista. Serie Técnica 12. 59 p. http://www.inta.gov.ar/bellavista/info/documentos/citricos/res.st12.htm

Ndou, P. y A. Obi (2011): The business environment and internacional competitiveness of Sout African citrus industry. 25 p. University of Fort Hare, South Africa. https://www.ifama.org/events/conferences/2011/cmsdocs/2011SymposiumDocs/352_Symposium \%20Paper.pdf

Porto, N. 2010. Economía Internacional. Trabajos Prácticos. Página web de la Cátedra. Universidad Nacional de la Plata. http://www.depeco.econo.unlp.edu.ar/ catedras/internac/pdfs/tp4.pdf

USDA (2010) EU 27 Citrus Annual 2010. Madrid. 20 p. http://gain.fas.usda.gov/Recent\%20GAIN\%20Publications/Citrus\%20Annual_Madrid_EU-27_12-152010.pdf Acceso a la página, Junio de 2011.

Zubrzycki, H. (1997). Calendario de producción de naranjas en las principales zonas citrícolas de Argentina, Boletín de Información Citrícola 9. Proyecto Diversificación Productiva. INTA Concordia. 10 p.

Base de Datos

FAOSTAT (2011), http://faostat.fao.org/

UN COMTRADE (2011) http://comtrade.un.org/, SENASA (2011): Oficina de Estadísticas de Comercio Exterior http://www.senasa.gov.ar/estadistica.php\# Trade Map (2011): Centro de Comercio Internacional: http://www.trademap.org/ 